\title{
Transient Grating Investigations at Liquid-Liquid Interfaces
}

\author{
Angela Punzi§, Pierre Brodard, and Eric Vauthey* \\ §SCS Poster Prize Winner
}

\begin{abstract}
A new four-wave-mixing technique with evanescent optical fields generated by total internal reflection at a liquid-liquid interface is described. Several applications of this method to measure thermoacoustic and dynamic properties near liquid-liquid interfaces are presented.
\end{abstract}

Keywords: Acoustic properties · Liquid-liquid interfaces · Orientational anisotropy ·

Transient grating spectroscopy $\cdot$ Ultrafast dynamics

Liquid-liquid interfaces (LLI) are involved in many areas of science and technology, such as chemical extraction and drug delivery [1]. Detailed information on the structure and the dynamics properties of LLI are thus required to fully understand these processes. Information on LLI is still extremely scarce because of the difficulty to experimentally access this region. Although the thickness of the LLI is still debated, it is generally accepted that it does not exceed a few nanometers. Therefore, if one performs conventional optical spectroscopy on such binary systems, the signal arising from the LLI is totally buried in that originating from the bulk phase.

There have been essentially two approaches to circumvent or at least minimize this problem. The first consists in measuring the second order nonlinear optical susceptibility which, in the dipolar approximation, is non-zero at the interface but vanishes in the centrosymmetric bulk phases. Second harmonic and sum frequency generations have

${ }^{*}$ Correspondence: Prof. E. Vauthey

University of Geneva

Sciences II

Department of Physical Chemistry

30, Quai Ernest-Ansermet

$\mathrm{CH}-1211$ Geneva 4

Tel: : +41223796537

Fax: + 41223796804

E-Mail: eric.vauthey@chiphy.unige.ch

http://www.unige.ch/sciences/chifi/Vauthey thus become major spectroscopic tools for interfaces [2][3]. One of the limitations of this approach is the impossibility to obtain a depth profile of the interfacial region. The second approach consists in confining the optical beam within a small volume close to the interface using evanescent optical fields generated for example upon total internal reflection (TIR). TIR fluorescence yields dynamic information on the LLI, but it is limited to emitting species [4].

Transient grating (TG) techniques are known to combine the high sensitivity of fluorescence, due to the zero-background nature of the signal, with that of transient absorption. Another advantage of the TG techniques is their sensitivity to photoinduced changes of refractive index [5].

We are developing TG techniques with evanescent waves to investigate the dynamics of photoinduced processes at LLI [6][7].

Fig. 1 illustrates the principle of a conventional TG experiment. Excitation of the sample by two time-coincident pump pulses leads to a spatial modulation of the light intensity, and upon interaction with the sample material, to a spatial modulation of the sample optical properties, namely of the refractive index and/or the absorbance. This transient holographic grating is then detected by a third time-delayed pulse at the probe wavelength, $\lambda_{p r}$, which strikes the grating at the Bragg angle, and is then partially diffracted. The diffracted intensity, $I_{d i f}$, can be expressed as:

$$
I_{d i f} \approx c_{1} \Delta n^{2}+c_{2} \Delta A^{2}
$$

where $c_{1}$ and $c_{2}$ are constants, and $\Delta n$ and $\Delta A$ are the modulation amplitudes of refractive index and absorbance, respectively. While $\Delta A$ is essentially due to population changes, $\Delta n$ can have several origins: population changes, $\Delta n_{p}$, density changes, $\Delta n_{d}$, and optical Kerr effect, $\Delta n_{O K E}$. As explained in more detail elsewhere [8], these different contributions can be distinguished by their different timescale and by their specific wavelength and polarization dependence. One important application of the TG techniques is the measurement of population dynamics [9]. For this purpose, the probe wavelength has to coincide with an absorption band associated with the investigated population. Moreover, to avoid any contribution due to the orientational anisotropy created by the two pump pulses, the polarization of the probe field must be at the magic angle (MA), $54.7^{\circ}$, relative to that of the pump pulses. In this case, the diffracted intensity is given by:

$$
I_{d i f}^{M A}(t) \propto \Delta C^{2}(t)
$$

where $\Delta C$ is the modulation amplitude of the concentration of the probed species.

On the other hand, excitation of the sample with orthogonally polarized pump pulses leads to a so-called polarization or crossed grating (CG). Light intensity on the crossing region is now uniform, but the polarization of the electric field is spatially modulated. After interaction with the sample material, spatial modulation of dichroism and birefringence is created. Probing such a grating with a probe pulses with the same polarization as one of the pump 


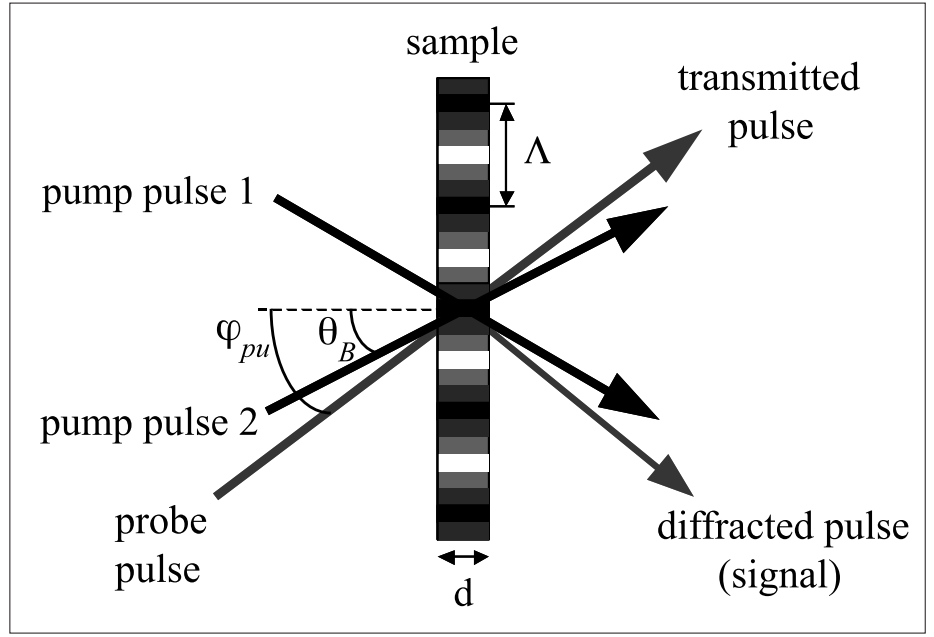

Fig. 1. Principle of the transient grating technique. $\varphi_{p u}$ and $\theta_{\mathrm{B}}$ are the angle of incidence of the two pump pulses and the Bragg angle, respectively

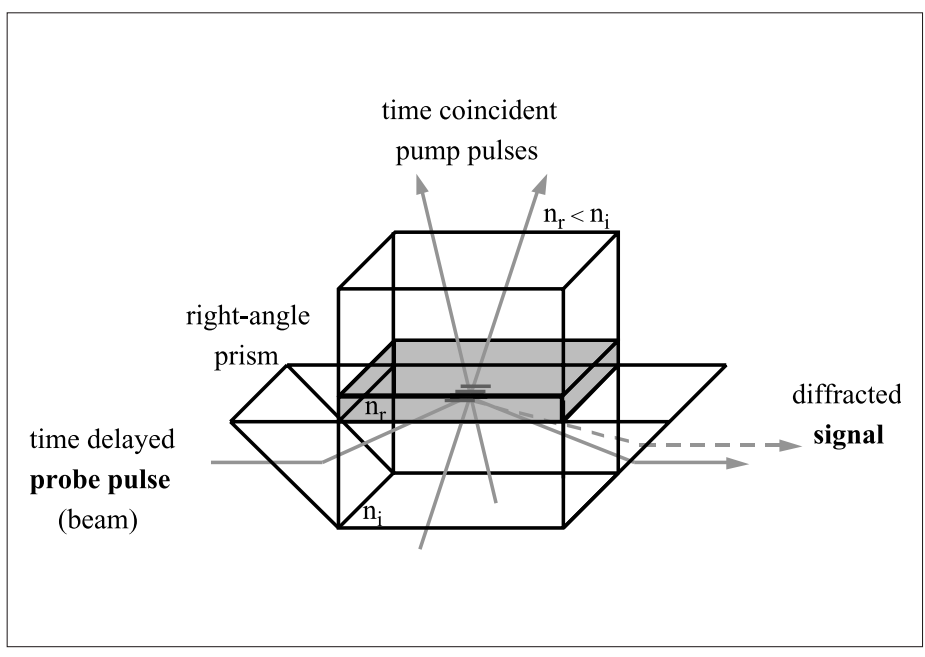

Fig. 2. Transient evanescent grating geometry with TIR-probe pulses, leads to a diffracted signal with the same polarization as the other pump pulse, the $\mathrm{CG}$ acting as a half-wave plate. In this case, the diffracted intensity is given by:

$$
I_{d i f}^{C G}(t) \propto(\Delta C(t) \cdot r(t))^{2}
$$

where $r$ is the orientational anisotropy. The two major mechanisms for the anisotropy decay are the rotational diffusion, which takes place in the sub-nanosecond timescale in non-viscous liquids, and excitation energy hopping, which occurs predominantly at high concentration of chromophores or in aggregates.

Finally, if the probe wavelength is far from any absorption band of the sample material (non-resonant probing, NR), the diffracted intensity is essentially given by:

$$
I_{d i f}^{N R} \propto\left(\Delta n_{d}+\Delta n_{O K E}\right)^{2}
$$

These two contributions can be very easily distinguished by their very different timescale, the OKE signal appearing around time zero, when the pump and probe pulses temporally overlap. On the other hand, the formation of the density grating after a heat releasing process, such as an internal conversion, is not instantaneous and is characterized by the acoustic period, $\tau_{a c}$, which depends on the fringe spacing, $\Lambda$, and on the speed of sound. If the timescale of the heat releasing process is much shorter than $\tau_{a c}$, the impulsive thermal expansion leads to the generation of acoustic waves, whose propagation leads to a temporal modulation of the diffracted intensity. The speed of sound in a material can be easily determined from such profiles.

Fig. 2 shows one of the three experimental arrangements used to investigate the dynamics of photoinduced processes near a LLI. The sample consists in a solution of rhodamine 6G $(\mathrm{R} 6 \mathrm{G})$ in methanol $(\mathrm{MeOH})$ in contact with decaline (DECA). A thick grating is produced in the dye solution by the pump pulses and interfacial selectivity is achieved by using an evanescent probe field, generated by TIR at the interface. The thickness of the probed layer is typically of the order of $\lambda_{p r} / 8$ to $\lambda_{p r} / 4$, depending on the angle of incidence. An improvement by a factor of two or three can be achieved by using evanescent pump and evanescent pumpprobe fields, respectively [7].

Fig. 3 shows the time profile of the diffracted intensity measured after excitation with two pump pulses at $532 \mathrm{~nm}$ in TIR and upon NR probing with a cw-laser beam at $800 \mathrm{~nm}$. The diffracted intensity is due to $\Delta n_{d}$ only, and the oscillations reflect the propagations of acoustic waves. The Fourier transform of the time profile (inset of Fig. 3) indicates the presence of two acoustic waves. As their wavelength is the same and is determined by the fringe spacing of the grating, it appears that one acoustic wave is propagating in $\mathrm{MeOH}$ and is due to thermal expansion (TE), while the other travels in DECA and is due to electrostriction (ES). ES is a non-resonant process, which leads to a material compression in the regions of high electric field strength, hence to the generation of an acoustic wave. In principle, the contribution of ES to $\Delta n_{d}$ is almost negligible but in the present geometry, the sample volume responsible for the resonant response is much smaller than the volume of pump and probe beam overlapping region in DECA. From such measurements, it was found that the speed of sound in $\mathrm{MeOH}$ close to the interface with DECA is not significantly different from that in the bulk solution [7][10]. Experiments have also been performed upon addition of a surfactant to the dye solution. In this case, the interfacial speed of sound was found to differ significantly from that measured in bulk dye solutions with surfactant. This differ- ence, which was ascribed to the adsorption of the surfactant at the interface [7][10], illustrates the interfacial selectivity of the TG techniques with evanescent waves.

Fig. 4 shows the time profile of the diffracted intensity measured after excitation with two pump pulses at $532 \mathrm{~nm}$ and probing with a $532 \mathrm{~nm}$ pulse in TIR, with the same system $\left([R 6 G]=2.9 \cdot 10^{-2} \mathrm{M}\right)$. The slow decaying time profile has been recorded using MA configuration and therefore reflects the ground state recovery dynamics (GSR) of R6G. The GSR time constant of 240 ps obtained here differs strongly from that of about $4 \mathrm{~ns}$ found in diluted R6G solutions [11]. Above $5 \cdot 10^{-3} \mathrm{M}$, this dye forms aggregates, which shorten the $S_{1}$ lifetime of the monomer by energy transfer quenching [12]. The ensuing excited aggregates decay non-radiatively in the ps timescale.

The measurements have also been carried out in bulk solutions with the same R6G concentrations. The GSR time constant was found to be about three times larger $\left(\tau_{G S R}\right.$ $=760 \mathrm{ps}$ ) than at the interface. Similar differences between bulk and interface have been observed at other concentrations ranging from $10^{-2}$ to $5 \cdot 10^{-2} \mathrm{M}$. This effect is ascribed to the adsorption of R6G at the interface, which leads to an enhancement of the local dye concentration in the probed region.

The fast decaying time profile shown in Fig. 4 was recorded using CG geometry. It is essentially identical to the response function of the experimental setup and indicates a decay of the orientational anisotropy faster than about 20 to $30 \mathrm{ps}$. Similar time profiles have been measured in bulk solutions with the same R6G concentrations. On the other hand the anisotropy decay time of a diluted R6G solution in $\mathrm{MeOH}$ amounts to 164 ps [11]. In this case, the anisotropy decays by reorientational diffusion of the $\mathrm{R} 6 \mathrm{G}$ molecules. The much shorter decay time measured at high concentrations can be as- 


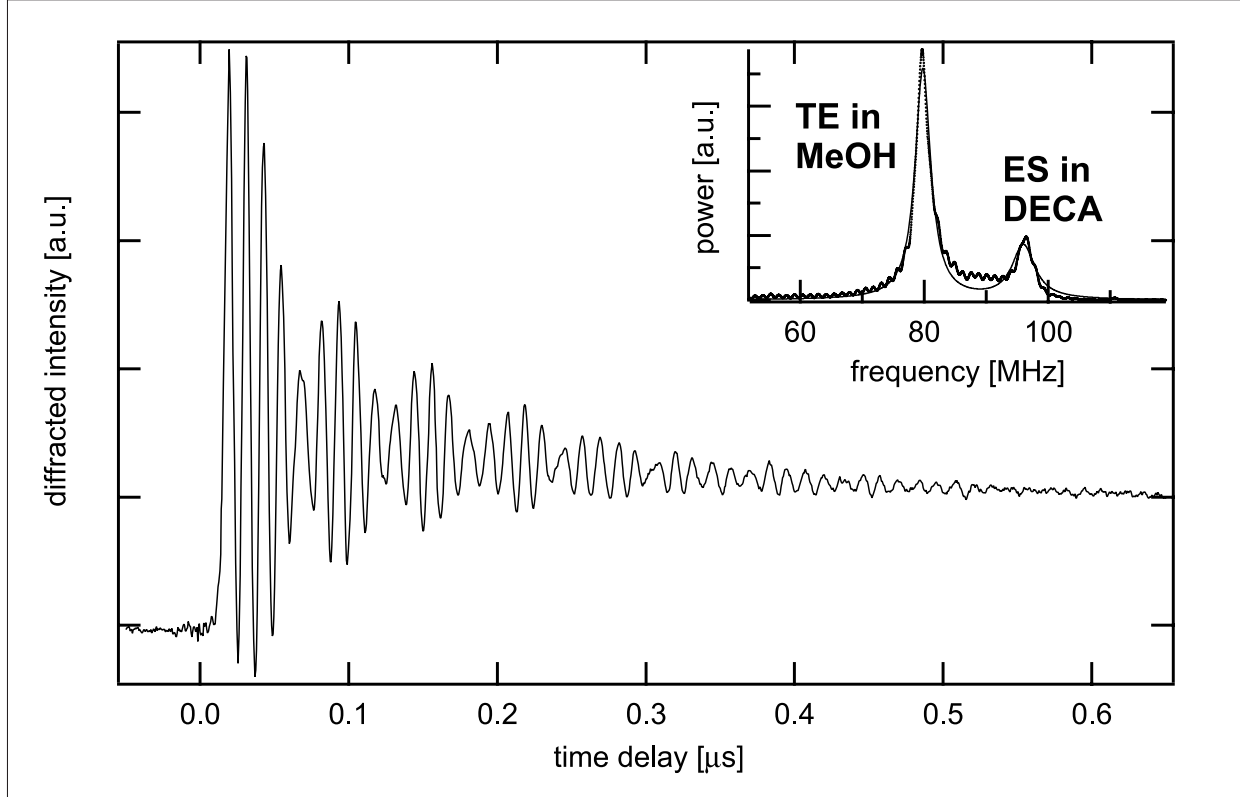

Fig. 3. Time profile of the diffracted intensity measured with a solution of R6G in $\mathrm{MeOH}$ in contact with DECA, using TIR-pump geometry and cw probing. Inset: power spectrum obtained upon Fourier transformation of the time profile.

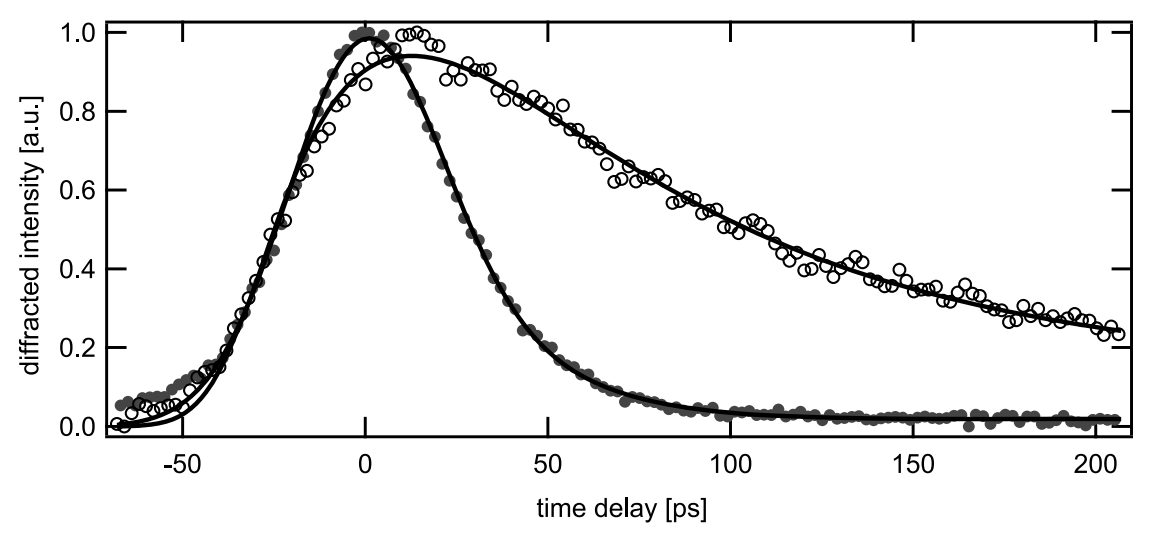

Fig. 4. Time profiles of the diffracted intensity measured with a solution of $\mathrm{R} 6 \mathrm{G}$ in $\mathrm{MeOH}([\mathrm{R} 6 \mathrm{G}]=$ $2.9 \times 10^{-2} \mathrm{M}$ ) in contact with DECA, using the TIR-probe geometry with MA (empty circles) and CG configuration (black dots)

signed to the opening of a new channel for anisotropy decay, most probably excitation energy hopping between R6G molecules. Energy hopping does not lead to quenching and therefore, the decay of the MA time profile, which reflects the R6G excited state population, is slower than that recorded in the CG geometry.

The comparison between MA and CG dynamics is particularly interesting, because the first reflects the energy transfer between monomeric R6G and aggregates, while the second is due to energy transfer between monomeric R6G molecules. The CG measurements will be repeated with shorter pulses to time resolve the energy hopping dynamics and to look for possible differences between bulk and interface. Moreover, in order to evaluate the interfa- cial selectivity of the transient evanescent grating techniques, similar systems will also be investigated by time-resolved second harmonic generation.

\section{Acknowledgements}

This work is supported by the Fonds National Suisse de la Recherche Scientifique through project 200020-100014.

Received: December 12, 2004

[1] 'Liquid interfaces in chemical, biological, and pharmaceutical applications', Ed. A.G. Volkov, Marcel Dekker, 2001.

[2] K.B. Eisenthal, Chem. Rev. 1996, 96, 1343.

[3] G.L. Richmond, Chem. Rev. 2002, 102, 2693.
[4] S. Ishizaka, N. Kitamura, Bull. Chem. Soc. Jpn. 2001, 74, 1983.

[5] H.J. Eichler, P. Günter, D.W. Pohl, in 'Laser-Induced Dynamic Gratings’, SpringerVerlag, Berlin, 1986.

[6] P. Brodard, E. Vauthey, Rev. Sci. Instrum. 2003, 74, 725.

[7] P. Brodard, PhD Thesis, University of Geneva, 2004.

[8] E. Vauthey, in 'Encyclopedia of Modern Optics' Eds. G.R. Steel, D.G. Bayvel, Elsevier: Oxford, 2004, Vol. 1, pp 73.

[9] C. Högemann, M. Pauchard, E. Vauthey, Rev. Sci. Instrum. 1996, 67, 3449.

[10] P. Brodard, E. Vauthey, J. Phys. Chim. B 2005, 109, in press.

[11] J.-C. Gumy, E. Vauthey, J. Phys. Chem. 1996, 100, 8628.

[12] A. Penzkofer, Y. Lu, Chem. Phys. 1986, 103, 399. 